\title{
Generalized ghost-free quadratic curvature gravity
}

\author{
Tirthabir Biswas ${ }^{1}$, Aindriú Conroy ${ }^{2}$, Alexey S. Koshelev ${ }^{3}$ and \\ Anupam Mazumdar ${ }^{2}$ \\ ${ }^{1}$ Department of Physics, Loyola University, New Orleans, LA 56302 \\ ${ }^{2}$ Consortium for Fundamental Physics, Lancaster University, Lancaster, LA1 4YB, \\ UK \\ 3 Theoretische Natuurkunde, Vrije Universiteit Brussel and The International Solvay \\ Institutes, Pleinlaan 2, B-1050, Brussels, Belgium \\ E-mail: tbiswas@loyno.edu, a.conroy@lancaster.ac.uk, \\ alexey.koshelev@vub.ac.be, a.mazumdar@lancaster.ac.uk
}

\begin{abstract}
In this paper we study the most general covariant action of gravity up to terms that are quadratic in curvature. In particular this includes non-local, infinite derivative theories of gravity which are ghost-free and exhibit asymptotic freedom in the ultraviolet. We provide a detailed algorithm for deriving the equations of motion for such actions containing an arbitrary number of the covariant D'Alembertian operators, and this is our main result. We also perform a number of tests on the field equations we derive, including checking the Bianchi identities and the weak-field limit. Lastly, we consider the special subclass of ghost and asymptotically free theories of gravity by way of an example.
\end{abstract}

PACS numbers: 98.80.Bp,11.10.Lm

Submitted to: Class. Quantum Grav. 


\section{Introduction}

Einstein's general relativity (GR) is a remarkable theory in many respects but there still remain some major unresolved questions. Despite a vast amount of observational data in favour of GR [1], the presence of cosmological and black hole singularities are examples of problems at the classical level which strongly suggest the incompleteness (or inconsistency) of GR in the ultraviolet (UV). At the quantum level GR is not UV complete. Modification of GR is demanded in this regard but one must take care of preserving the conformance with the available data. Indeed, the above mentioned classical and quantum problems could be closely related as both concern short-distance physics.

Most of the work on modifying GR has concentrated upon studying finite higher order (derivative) gravity such as "Fourth Order Gravity" which are quadratic in curvatures:

$$
\mathcal{L} \sim R+f_{1} R^{2}+f_{2} R_{\mu \nu} R^{\mu \nu}+f_{3} R^{\mu \nu \lambda \sigma} R_{\mu \nu \lambda \sigma}
$$

where $\mu, \nu=0,1,2,3$ and $f_{1}, f_{2}, f_{3}$ are appropriate constant coefficients - a particular variant of which give the famous Gauss-Bonnet gravity [2].

The above action naturally yields 4 derivatives and it was studied extensively by Stelle in Refs. [3, 4, 5]. One of the most interesting properties of 4th derivative gravity is that it can be made renormalizable [3], thus in the UV the modified graviton propagator leads to convergence of the Feynman diagrams at 1-loop and beyond. However, this comes at the cost of the presence of the "Weyl" ghost in the tensor component of the modified graviton propagator [3].

The authors Biswas, Mazumdar and Siegel (BMS) in Ref. [6] argued that the absence of ghosts in the modified propagator coupled with asymptotic freedom can only be realized if one considers an infinite set of higher derivative terms, in particular in the form of an exponential, that are allowed by general covariance, see also [7]. Although, the infinite derivative action considered in [6] was only partially successful in realizing asymptotic freedom, it was able to find cosmological non-singular (without the Big Crunch/Bang singularity) bouncing solutions in these type of theories. Based on the action of BMS, cosmological perturbation analyses were performed in Refs. [8] which further demonstrate the robustness of the bouncing model and its possible connection to inflationary cosmologyt. All this strongly suggests that it may be possible to weaken gravitational interactions in a consistent manner at short distances and at early times.

Indeed, recently it was shown by Biswas, Gerwick, Koivisto and Mazumdar (BGKM) in Ref. [10] that gravity in the UV can be made asymptotically free without violating basic principles of physics, such as unitarity and general covariance by including appropriate infinite set of higher derivative terms. The action considered in Ref. [10] is

$\ddagger$ For cosmological applications of infinite derivative scalar field theories, see for instance [9] and references therein. 
of the form

$S=\int d^{4} x \sqrt{-g}\left(\frac{R}{2}+R \mathcal{F}_{1}(\square) R+R^{\mu \nu} \mathcal{F}_{2}(\square) R_{\mu \nu}+C^{\mu \nu \lambda \sigma} \mathcal{F}_{3}(\square) C_{\mu \nu \lambda \sigma}\right)$,

where $\mathcal{F}_{i}(\square)$ are functions of the D'Alembertian operator, $\square=g^{\mu \nu} \nabla_{\mu} \nabla_{\nu}, \nabla_{\nu}$ is the covariant derivative and $g_{\mu \nu}$ is 4 dimensional metric with $(-,+,+,+), R_{\mu \nu}$ and $C_{\mu \nu \lambda \sigma}$ are the Ricci and Weyl tensors $\S$ respectively which are given in terms of the Riemann tensor, $R_{\mu \nu \lambda \sigma}$, as usual via

$$
C_{\alpha \nu \beta}^{\mu}=R_{\alpha \nu \beta}^{\mu}-\frac{1}{2}\left(\delta_{\nu}^{\mu} R_{\alpha \beta}-\delta_{\beta}^{\mu} R_{\alpha \nu}+R_{\nu}^{\mu} g_{\alpha \beta}-R_{\beta}^{\mu} g_{\alpha \nu}\right)+\frac{R}{6}\left(\delta_{\nu}^{\mu} g_{\alpha \beta}-\delta_{\beta}^{\mu} g_{\alpha \nu}\right)(2)
$$

We have also set the reduced Planck mass to one, and we only consider $\mathcal{F}_{i}(x)$ 's that are analytic at $x=0$, otherwise one does not recover GR in the infrared, see [10] for details. Further analytic stable cosmological bouncing solutions for action (1) were found in [11.

Gravity being a gauge theory, i.e. diffeomorphism invariant, should allow all possible gauge invariant terms in its "effective action" and (11), at least, contains all possible terms with at most two curvatures. We emphasize that $\mathcal{F}_{i}(x)$ 's can be transcendental functions containing an infinite set of derivatives and this is of course the case that is of most interest to us. We should point out that there has been a growing interest in recent years in such/similar infinite derivative gravitational actions deriving its motivation from cosmology [12], renormalization group flows [13] and quantum gravity [14]. We also find it curious to note that such infinite higher derivative actions appear in non-perturbative string theories, see for instance Ref. [15]. The higher derivative corrections essentially take into account the $\alpha^{\prime}$ corrections in string theory. Unfortunately, however, close string theories containing graviton physics do not yet provide an action with all orders in $\alpha^{\prime}$.

Let us now briefly expound upon the advantages of having an infinite set of derivatives. In GR the graviton propagator becomes

$$
\Pi_{G R} \sim \frac{P_{2}}{k^{2}}-\frac{P_{s}}{2 k^{2}} .
$$

where $P_{2}$ and $P_{s}$ refers to the projection operators corresponding to the massless spin-2 and the scalar degree of freedom in the metric, see Ref. [16] for details. The graviton propagator in BGKM extension (1) of gravity modifies to [10, 17]

$$
\Pi_{B G K M} \sim \frac{1}{f\left(k^{2}\right)}\left(\frac{P_{2}}{k^{2}}-\frac{P_{s}}{2 k^{2}}\right), \text { where } f\left(k^{2}\right) \rightarrow 1 \text { when } k^{2} \rightarrow 0 .
$$

In order for the propagator to be ghost-free, $f\left(k^{2}\right)$ must have no zeros on the complex plan£ $\llbracket$, so that there are no extra poles in the propagator. However, at low energies,

$\S$ In the original action introduced in Ref. [10, we had the Riemann tensor rather than the Weyl tensor. The Weyl tensor however is identically zero on conformally flat manifolds and therefore it is a more convenient choice for most calculations.

\| Such functions can be represented as the exponent of an entire function. For a polynomial $f\left(k^{2}\right)$ extra poles appear, an extra degree of freedom other than the massless graviton, and one generates new 
$k^{2} \rightarrow 0$, the above equation (44) asymptotes to the GR propagator, i.e. (3). An example of $f\left(k^{2}\right)$ which leads to a ghost and asymptotically free theory is given by [10, 17]

$$
f\left(k^{2}\right)=e^{\gamma k^{2}}
$$

with $\gamma>0$. The propagator is exponentially suppressed in the UV leading to softening of the gravitational force at short distance.

So far, based on linearized equations [10], we have found that non-local asymptotically free actions can indeed resolve black hole singularities, although the

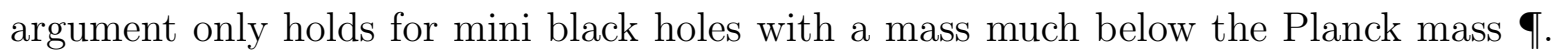
Further, the theory also admits cyclic cosmologies with nonsingular transitions from expansions to contractions (turnarounds) and vice versa (bounces). However, to study the robustness of the resolutions of these classical singularities one needs to go beyond the linearized equations of motion. This is the primary motivation for the present work where we obtain the field equations for the most general higher derivative action containing at most two curvatures, and generalize the results presented in [6, 8, 11]. A topical application of this would be to improve on Starobinsky's model of inflation [20], for a review on primordial inflation see [21], where only

$$
\mathcal{L} \sim R+R^{2}
$$

terms were considered, but clearly there is no reason why other quadratic curvature terms would be missing! For example, no underlying symmetry is known which could prevent these terms from appearing in the "effective action", and therefore one should really consider an action of the form (1). Actually, including such terms can lead to interesting phenomenological consequences: since such modifications are expected to produce bounce with a solution $a(t)=a_{0} \cosh (\kappa t)$, where $a_{0}$ and $\kappa$ are positive constants [6, 8, 11], which inevitably entail a super-inflationary phase near the bounce, such pre-inflationary dynamics may be able to account for the low multipoles that has been observed in CMB [22].

For most part, our paper is technical and we provide a detailed account of the methodology we used in taming actions such as (1). In fact, our algorithm for dealing with higher derivative actions involving an arbitrary number of $\square$ operators can be applied more generally to any action of the form

$$
S=\int d^{4} x S \mathcal{F}(\square) T
$$

where $S$ and $T$ are any tensors constructed out of the Riemann curvatures and the metric. We note in passing that over the last decade or so higher derivative gravitational degrees of freedom also in the equation of motion, which due to Ostrogradski analysis will be ghost-like in nature [18. For higher derivative theories the initial condition problem has also been addressed in Ref. [19].

I Similar argument holds for linearized gravitational waves in GR, where the aptitude of the gravitational wave diverges near the source, but with BGKM propagator the amplitude of the gravitational waves remain finite near the source throughout the domain while recovering that of the GR's prediction in the IR [10. 
actions have gained a lot of attention as alternatives to the dark energy paradigm (for a review see [23]), and some of the general techniques/results in our paper may be relevant for such investigations.

Our paper is organized as follows: Section 2 is devoted to deriving the field equations for (11). Section 3 deals with various checks of our results. Firstly, we verify that our results reproduce the field equations obtained in previous literature for some special cases. Secondly, we check that the Bianchi identities are satisfied. Although a somewhat arduous task, this is a strong indicator of the validity of our derivation; and finally we reduce our result in the weak-field limit by looking at perturbations around Minkowski space-time and analysing the Newtonian potentials and comparing with known expressions. In Section 4, we consider a particular subclass of quadratic curvature theories of gravity that are both ghost and asymptotically free, before concluding by summarizing our results and providing a brief outlook of possible future research. Finally, in the appendices, we give explicit details of the derivations of the equation of motion (Appendix A), the details of the Bianchi identity calculation (Appendix B) and the equation of motion in a form useful for comparison with sixth order gravity [24] (Appendix C).

\section{Action and equations of Motion}

Let us start by recalling that the most general generally-covariant gravity action with at most quadratic curvature terms is of the form [10]

$$
S_{q}=\int d^{4} x \sqrt{-g} R_{\mu_{1} \nu_{1} \lambda_{1} \sigma_{1}} \mathcal{O}_{\mu_{2} \nu_{2} \lambda_{2} \sigma_{2}}^{\mu_{1} \nu_{1} \lambda_{1} \sigma_{1}} R^{\mu_{2} \nu_{2} \lambda_{2} \sigma_{2}}
$$

As discussed in [10] we need only the graviton propagator to understand both the asymptotic behaviour of gravity in the UV and the potential problem with ghosts. The focus of [10] was on linearized solutions of gravity around the Minkowski background

$$
g_{\mu \nu}=\eta_{\mu \nu}+h_{\mu \nu}
$$

and accordingly one only needed to consider an action that contained terms which are quadratic in $h_{\mu \nu}$.

We now want to expand our treatment to include fluctuations around an arbitrary background for the most general quadratic curvature gravity action given by (1). For simplicity we often omit hereafter the explicit dependence of $\mathcal{F}_{i}(\square)$ on $\square$ writing simply $\mathcal{F}_{i}$.

\subsection{General Methodology}

2.1.1. Single $\square$ In order to elucidate the methods involved in finding the field equations, we will start with a simple example:

$$
S_{p}=\int d^{4} x \sqrt{-g} T \square S
$$


where $S$ and $T$ are arbitrary scalars made out of the Riemann and the metric tensors. Varying this gives us

$$
\delta S_{p}=\int d^{4} x \sqrt{-g}\left(\frac{h}{2} T \square S+\delta T \square S+T \delta(\square) S+T \square \delta S\right) .
$$

where we have defined

$$
\delta g_{\mu \nu}=h_{\mu \nu}
$$

so that +

$$
\delta g^{\mu \nu}=-h^{\mu \nu} .
$$

Variations of the various curvatures, such as $\delta R, \delta R_{\mu \nu}$ and $\delta C_{\mu \nu \lambda \sigma}$ are well known and are given in Appendix A for completeness. Any composite function of these can be straightforwardly computed with the help of the individual variations. Calculation of $\delta(\square) S$, however, is a little trickier and we will provide it below. The derivation of $\delta(\square) S_{\mu \nu}$ and $\delta(\square) S_{\mu \nu \lambda \sigma}$, which we will also need, can be found in Appendix A as well.

From the definition of the D'Alembertian operator we have

$$
\delta(\square) S=-h_{\alpha \beta} S^{; \alpha ; \beta}+g^{\mu \nu} \delta\left(\nabla_{\mu}\right) S_{; \nu}+g^{\mu \nu}\left[\delta\left(\nabla_{\nu}\right) S\right]_{; \mu},
$$

where semicolons denote the covariant derivative. Next, by using the general definition of the covariant derivative of a tensor and treating $S_{; \alpha}$ as a $(0,1)$-tensor one finds that

$$
g^{\mu \nu} \delta\left(\nabla_{\mu}\right) S_{; \nu}=-g^{\mu \nu} \delta \Gamma_{\mu \nu}^{\lambda} S_{; \lambda} .
$$

The trick is to observe that the $\delta$-variation operates only on the Christoffel symbols and not upon $S$ and in this case the final term vanishes as $S$ and $T$ are scalars. We then arrive at an important result:

$$
\delta(\square) S=-h_{\alpha \beta} S^{; \alpha ; \beta}+\frac{1}{2} g^{\alpha \beta} S_{; \lambda}\left(h_{\alpha \beta}\right)^{; \lambda}-S^{; \alpha}\left(h_{\alpha \beta}\right)^{; \beta} .
$$

The variation of the $S_{p}$ can now be written in a convenient form

$$
\delta S_{p}=\int d^{4} x \sqrt{-g}\left(g^{\mu \nu} T \square S+\square S \frac{\delta T}{\delta g_{\mu \nu}}+\square T \frac{\delta S}{\delta g_{\mu \nu}}\right) h_{\mu \nu}
$$

where we have liberally used integration by parts to transfer the covariant derivatives acting on $h_{\mu \nu}$ to $S, T$.

2.1.2. Multiple $\square$ 's What is crucial, of course, is that the above manipulations can be straight forwardly generalized to the case when one has many $\square$ operators. In particular, by repeated integration by parts one can show that the result in equation (14) is useful for all powers of the D'Alembertian operator and indeed any function $\mathcal{F}_{i}(\square)$. We have

$$
\int d^{4} x \sqrt{-g} T \delta\left(\square^{n}\right) S=\sum_{m=0}^{n-1} \int d^{4} x \sqrt{-g} \square^{m} T \delta(\square) \square^{n-m-1} S,
$$

\footnotetext{
+ The indices are always raised and lowered with respect to the background metric $g_{\mu \nu}$.
} 
which is itself simply a result of repeated integration by parts. Its equivalent in terms of an arbitrary function $\mathcal{F}_{i}(\square)$ is as follows

$$
\int d^{4} x \sqrt{-g} T \delta F_{i}(\square) S=\sum_{n=1}^{\infty} \sum_{m=0}^{n-1} \int d^{4} x \sqrt{-g} f_{i_{n}} \square^{m} T \delta(\square) \square^{n-m-1} S,
$$

where $f_{i_{n}}$ 's are the Taylor series expansion coefficients:

$$
\mathcal{F}_{i}(\square)=\sum_{n=0}^{\infty} f_{i_{n}} \square^{n}
$$

The important point is that $\square^{n-m-1} S$ is also a scalar, and therefore the way the $\delta \square$ operator acts on it is exactly the same as its action on $S$, one simply has to substitute $S \rightarrow \square^{n-m-1} S$ in (12). This is why, as we shall soon see, it becomes possible to calculate the variation of any term of the form

$$
\int d^{4} x \sqrt{-g} R \square^{n} R
$$

that appears in (1).

Moreover, one can use very similar methods to obtain variations such as $\delta(\square) \square^{n} R_{\mu \nu}$ and $\delta(\square) \square^{n} C_{\mu \nu \lambda \sigma}$; since the $\square$ operator does not affect the tensorial structure, all one needs is to find the general form of quantities such as $\delta(\square) S_{\mu \nu}$ and $\delta(\square) S_{\mu \nu \lambda \sigma}$. Also note, (17) actually holds for any $S$ and $T$ irrespective of their tensorial indices. Thus, one can adopt exactly the same procedure as for the scalar $S, T$ 's to obtain the variations involving the Ricci and Weyl tensors. Below we enumerate the somewhat cumbersome expressions for $\delta(\square) S_{\mu \nu}$ and $\delta(\square) S_{\mu \nu \lambda \sigma}$. The details of the derivations can be found in Appendix A.

$$
\begin{gathered}
\delta(\square) S_{\mu \nu}=-h_{\alpha \beta} S_{\mu \nu}^{; \alpha ; \beta}-\left(h_{\alpha \beta}\right)^{; \beta} S_{\mu \nu}^{; \alpha}+\frac{1}{2} g^{\alpha \beta}\left(h_{\alpha \beta}\right)^{; \sigma} S_{\mu \nu ; \sigma} \\
-\frac{1}{2}\left[\square\left(h_{\alpha \beta}\right) \delta_{(\mu}^{\beta} S_{\nu)}^{\alpha}-\left(h_{\alpha \beta}\right)^{; \tau ; \alpha} \delta_{(\mu}^{\beta} S_{\tau \nu)}+\left(h_{\alpha \beta}\right)_{;(\mu}^{; \beta} S_{\nu)}^{\alpha}\right] \\
\left.-S_{(\nu ; \beta}^{\alpha ; \beta} h_{\alpha \beta ; \mu)}-\delta_{(\mu}^{\beta} S_{\nu)}^{\alpha ; \lambda} h_{\alpha \beta ; \lambda}+\delta_{(\mu}^{\beta} S_{\tau \nu)}^{; \alpha} h_{\alpha \beta}^{; \tau}\right), \\
\delta(\square) S_{\mu \nu \lambda \sigma}=-h_{\alpha \beta} S_{\mu \nu \lambda \sigma}^{; \alpha ; \beta}-\left(h_{\alpha \beta}\right)^{; \beta} S_{\mu \nu \lambda \sigma}^{; \alpha}+\frac{1}{2} h^{; \tau} S_{\mu \nu \lambda \sigma ; \tau} \\
-\frac{1}{2}\left[g^{\alpha \tau}\left(h_{\alpha \beta}\right)_{; \mu}^{; \beta} S_{\tau \nu \lambda \sigma}+g^{\alpha \tau}\left(h_{\alpha \beta}\right)_{; \nu}^{; \beta} S_{\mu \tau \lambda \sigma}+g^{\alpha \tau}\left(h_{\alpha \beta}\right)_{; \lambda}^{; \beta} S_{\mu \nu \tau \sigma}+g^{\alpha \tau}\left(h_{\alpha \beta}\right)_{; \sigma}^{; \beta} S_{\mu \nu \lambda \tau}\right] \\
-\left[g^{\alpha \tau}\left(h_{\alpha \beta}\right)_{; \mu} S_{\tau \nu \lambda \sigma}^{; \beta}+g^{\alpha \tau}\left(h_{\alpha \beta}\right)_{; \nu} S_{\mu \tau \lambda \sigma}^{; \beta}+g^{\alpha \tau}\left(h_{\alpha \beta}\right)_{; \lambda} S_{\mu \nu \tau \sigma}^{; \beta}+g^{\alpha \tau}\left(h_{\alpha \beta}\right)_{; \sigma} S_{\mu \nu \lambda \tau}^{; \beta}\right] .
\end{gathered}
$$

\subsection{Variation of the Quadratic Curvature Action}

We are now ready to compute the variation of our action (11) and obtain the field equations. Let us define the "gravitational energy-momentum tensor" as

$$
P^{\alpha \beta}=-\frac{2}{\sqrt{-g}} \frac{\delta S}{\delta g_{\alpha \beta}}=-\frac{2}{\sqrt{-g}} \frac{\delta S}{h_{\alpha \beta}} .
$$


We shall compute the contribution to $P^{\alpha \beta}$ for the individual terms in the action (1) separately. We direct the reader to (52) for the complete field equations, should they wish to skip the technical details.

2.2.1. Computing $\delta S_{0} \quad S_{0}$ is nothing more than the Einstein-Hilbert action and it's variation is well known:

$$
P_{0}^{\alpha \beta}=G^{\alpha \beta}=-\frac{2}{\sqrt{-g}} \frac{\delta S_{0}}{h_{\alpha \beta}},
$$

where $G^{\alpha \beta}=R^{\alpha \beta}-\frac{1}{2} g^{\alpha \beta} R$ is the Einstein tensor.

2.2.2. Computing $\delta S_{1}$ The next step is to compute the variation of

$$
S_{1}=\int d^{4} x \sqrt{-g} R \mathcal{F}_{1}(\square) R
$$

Varying this and substituting values for $\delta R$ and $\delta \sqrt{-g}$ we find after appropriate integration by parts

$$
\begin{aligned}
\delta S_{1}= & \int d^{4} x \sqrt{-g}\left(\frac{1}{2} g^{\alpha \beta} R \mathcal{F}_{1} R+2\left(\mathcal{F}_{1} R\right)^{; \alpha ; \beta}-2 g^{\alpha \beta} \square\left(\mathcal{F}_{1} R\right)\right. \\
& \left.-2 R^{\alpha \beta} \mathcal{F}_{1} R\right) h_{\alpha \beta}+\sqrt{-g} R \delta \mathcal{F}_{1} R .
\end{aligned}
$$

To calculate the final term, we must employ the identity in (17). This gives us

$$
R \delta \mathcal{F}_{1}(\square) R=\sum_{n=1}^{\infty} \sum_{m=0}^{n-1} f_{i_{n}} R^{(m)} \delta(\square) R^{(n-m-1)},
$$

where $R^{(m)} \equiv \square^{m} R$ and the analogous notation is used hereafter for any tensor. We then substitute our value for $\delta(\square)$ and integrate by parts. Further terms will cancel by noting that

$$
\int d^{4} x \sqrt{-g} \sum_{n=1}^{\infty} \sum_{m=0}^{n-1} A^{(m)} B^{(n-m-1)}=\int d^{4} x \sqrt{-g} \sum_{n=1}^{\infty} \sum_{m=0}^{n-1} A^{(n-m-1)} B^{(m)},
$$

until we arrive at the energy-momentum tensor contribution:

$$
\begin{aligned}
P_{1}^{\alpha \beta}= & 4 G^{\alpha \beta} \mathcal{F}_{1}(\square) R+g^{\alpha \beta} R \mathcal{F}_{1}(\square) R-4\left(\nabla^{\alpha} \nabla^{\beta}-g^{\alpha \beta} \square\right) \mathcal{F}_{1}(\square) R \\
& -2 \Omega_{1}^{\alpha \beta}+g^{\alpha \beta}\left(\Omega_{1 \sigma}^{\sigma}+\bar{\Omega}_{1}\right),
\end{aligned}
$$

with

$$
\Omega_{1}^{\alpha \beta}=\sum_{n=1}^{\infty} f_{1_{n}} \sum_{l=0}^{n-1} \nabla^{\alpha} R^{(l)} \nabla^{\beta} R^{(n-l-1)}, \quad \bar{\Omega}_{1}=\sum_{n=1}^{\infty} f_{1_{n}} \sum_{l=0}^{n-1} R^{(l)} R^{(n-l)},
$$

and $P_{1}^{\alpha \beta}$ is defined via

$$
P_{1}^{\alpha \beta}=-\frac{2}{\sqrt{-g}} \frac{\delta S_{1}}{h_{\alpha \beta}} .
$$


2.2.3. Computing $\delta S_{2}$ We now focus on

$$
S_{2}=\int d^{4} x \sqrt{-g}\left(R^{\mu \nu} \mathcal{F}_{2}(\square) R_{\mu \nu}\right)
$$

Varying the action, we find

$$
\begin{aligned}
\delta S_{2}= & \int d^{4} x \sqrt{-g}\left[\frac{1}{2} g^{\alpha \beta} R^{\mu \nu} \mathcal{F}_{2} R_{\mu \nu}-2 R_{\mu}^{\beta} \mathcal{F}_{2} R^{\mu \alpha}+2\left(\mathcal{F}_{2} R^{\mu \beta}\right)_{; \mu}^{; \alpha}\right. \\
& \left.-\square\left(\mathcal{F}_{2} R^{\alpha \beta}\right)-g^{\alpha \beta}\left(\mathcal{F}_{2} R^{\mu \nu}\right)_{; \mu ; \nu}-\frac{1}{2} R \mathcal{F}_{2} R^{\alpha \beta}\right] h_{\alpha \beta} \\
& +\int d^{4} x \sqrt{-g} R_{\mu \nu} \delta \mathcal{F}_{2} R^{\mu \nu} .
\end{aligned}
$$

To compute the final term, we employ the method outlined in the previous Subsection and in particular (20), here we reiterate the main steps. Using identity (17) we have

$$
\sum_{n=1}^{\infty} f_{2_{n}} R^{\mu \nu} \delta\left(\square^{n}\right) R_{\mu \nu}=\sum_{m=0}^{n-1} \sum_{n=1}^{\infty} f_{2_{n}} R^{\mu \nu(m)} \delta(\square) R_{\mu \nu}^{(n-m-1)} .
$$

Then using (201) to compute $\delta(\square) R_{\mu \nu}^{(n-m-1)}$ and integrating by parts we find

$$
\begin{aligned}
& \int d^{4} x \sqrt{-g} R^{\mu \nu} \delta \mathcal{F}_{2}(\square) S_{\mu \nu}=\int d^{4} x \sqrt{-g} \sum_{m=0}^{n-1} \sum_{n=1}^{\infty} f_{2_{n}}\left[-S^{\mu \nu(m)} S_{\mu \nu}^{(n-m-1) ; \alpha ; \beta}\right. \\
& +\left[S^{\mu \nu(m)} S_{\mu \nu}^{(n-m-1) ; \alpha}\right]^{; \beta}-\frac{1}{2} g^{\alpha \beta}\left[S^{\mu \nu(m)} S_{\mu \nu ; \sigma}^{(n-m-1)}\right] ; \sigma \\
& -\frac{1}{2}\left[S^{\mu \nu(m)} S_{(\nu}^{\alpha(n-m-1)}\right]_{; \mu)}^{; \beta}-\frac{1}{2} \square\left[S^{\mu \nu(m)} \delta_{(\mu}^{\beta} S_{\nu)}^{\alpha(n-m-1)}\right] \\
& +\frac{1}{2}\left[S^{\mu \nu(m)} \delta_{(\mu}^{\beta} S_{\sigma \nu)}^{(n-m-1)}\right] ; \sigma ; \alpha+\left[S^{\mu \nu(m)} S_{(\nu}^{\alpha(n-m-1) ; \beta}\right]_{; \mu)} \\
& +\left[S^{\mu \nu(m)} \delta_{(\mu}^{\beta} S_{\nu)}^{\alpha(n-m-1) ; \sigma}\right]_{; \sigma}-\left[S^{\mu \nu(m)} \delta_{(\mu}^{\beta} S_{\sigma \nu}^{(n-m-1) ; \alpha] ; \sigma}\right] h_{\alpha \beta} .
\end{aligned}
$$

Changing the summation order we can reduce significantly the clutter so that for the Ricci tensor $R_{\mu \nu}$ we find

$$
\int d^{4} x \sqrt{-g} R^{\mu \nu} \delta \mathcal{F}_{2}(\square) R_{\mu \nu}=\int d^{4} x \sqrt{-g}\left(\Omega_{2}^{\alpha \beta}-\frac{1}{2} g^{\alpha \beta}\left(\Omega_{2 \sigma}^{\sigma}+\bar{\Omega}_{2}\right)+2 \Delta_{2}^{\alpha \beta}\right) h_{\alpha \beta},
$$

which combined with (32), gives us the energy-momentum component

$$
\begin{gathered}
P_{2}^{\alpha \beta}=-g^{\alpha \beta} R^{\mu \nu} \mathcal{F}_{2}(\square) R_{\mu \nu}+4 R_{\mu}^{\beta} \mathcal{F}_{2}(\square) R^{\mu \alpha}-4\left(\mathcal{F}_{2}(\square) R^{\mu \beta}\right)_{; \mu}^{; \alpha}+2 \square\left(\mathcal{F}_{2}(\square) R^{\alpha \beta}\right) \\
+2 g^{\alpha \beta}\left(\mathcal{F}_{2}(\square) R^{\mu \nu}\right)_{; \mu ; \nu}-2 \Omega_{2}^{\alpha \beta}+g^{\alpha \beta}\left(\Omega_{2 \sigma}^{\sigma}+\bar{\Omega}_{2}\right)-4 \Delta_{2}^{\alpha \beta} .
\end{gathered}
$$

Here

$$
\begin{aligned}
& \Omega_{2}^{\alpha \beta}=\sum_{n=1}^{\infty} f_{2_{n}} \sum_{l=0}^{n-1} R_{\nu}^{\mu ; \alpha(l)} R_{\mu}^{\nu ; \beta(n-l-1)}, \quad \bar{\Omega}_{2}=\sum_{n=1}^{\infty} f_{2_{n}} \sum_{l=0}^{n-1} R_{\nu}^{\mu(l)} R_{\mu}^{\nu(n-l)}, \\
& \Delta_{2}^{\alpha \beta}=\frac{1}{2} \sum_{n=1}^{\infty} f_{2_{n}} \sum_{l=0}^{n-1}\left[R_{\sigma}^{\nu(l)} R^{(\beta|\sigma| ; \alpha)(n-l-1)}-R_{\sigma}^{\nu ;(\alpha(l)} R^{\beta) \sigma(n-l-1)}\right]_{; \nu} .
\end{aligned}
$$


2.2.4. Computing $\delta S_{3}$ Finally we focus on the terms involving the Weyl tensors:

$$
S_{3}=\int d^{4} x \sqrt{-g}\left(C^{\mu \nu \lambda \sigma} \mathcal{F}_{3}(\square) C_{\mu \nu \lambda \sigma}\right)
$$

Varying the action we find

$\delta S_{3}=\int d^{4} x \frac{1}{2} \sqrt{-g} g^{\alpha \beta} h_{\alpha \beta}\left(C^{\mu \nu \lambda \sigma} \mathcal{F}_{3}(\square) C_{\mu \nu \lambda \sigma}\right)+\sqrt{-g} \delta\left(C^{\mu \nu \lambda \sigma} \mathcal{F}_{3}(\square) C_{\mu \nu \lambda \sigma}\right)$.

Now we find the first term develops as follows

$$
\begin{array}{r}
\delta\left(C^{\mu \nu \lambda \sigma} \mathcal{F}_{3} C_{\mu \nu \lambda \sigma}\right)=\delta C^{\mu \nu \lambda \sigma} \mathcal{F}_{3} C_{\mu \nu \lambda \sigma}+C^{\mu \nu \lambda \sigma} \mathcal{F}_{3} \delta C_{\mu \nu \lambda \sigma}+C^{\mu \nu \lambda \sigma} \delta \mathcal{F}_{3} C_{\mu \nu \lambda \sigma} \\
=-4 C_{\rho \theta \psi}^{\alpha} \mathcal{F}_{3} C^{\beta \rho \theta \psi} h_{\alpha \beta}+2 C^{\mu \nu \lambda \sigma} \mathcal{F}_{3} \delta C_{\mu \nu \lambda \sigma}+C^{\mu \nu \lambda \sigma} \delta \mathcal{F}_{3} C_{\mu \nu \lambda \sigma}
\end{array}
$$

Next, using the definition of the Weyl tensor (2), we note that

$$
\begin{aligned}
& 2 \delta C_{\alpha \nu \beta}^{\mu} \mathcal{F}_{3} C_{\mu}^{\alpha \nu \beta}=2\left[\delta R_{\alpha \nu \beta}^{\mu}-\frac{1}{2}\left(R_{\nu}^{\mu} h_{\alpha \beta}-R_{\beta}^{\mu} h_{\alpha \nu}\right)\right] \mathcal{F}_{3} C_{\mu}^{\alpha \nu \beta} \\
& =2\left[\delta R_{\alpha \nu \beta}^{\mu}-R_{\nu}^{\mu} h_{\alpha \beta}\right] \mathcal{F}_{3} C_{\mu}^{\alpha \nu \beta},
\end{aligned}
$$

paying careful attention when lowering the indices of the varied Weyl tensor. From Appendix A we have the variation of the Riemann tensor

$$
\delta R_{\alpha \nu \beta}^{\mu}=\frac{1}{2}\left[\delta_{\nu}^{\sigma} \delta_{\alpha}^{\tau}\left(h_{\sigma \tau}\right)_{; \beta}^{; \mu}-\delta_{\nu}^{\sigma} g^{\mu \tau}\left(h_{\sigma \tau}\right)_{; \beta ; \alpha}+g^{\mu \sigma} \delta_{\beta}^{\tau}\left(h_{\sigma \tau}\right)_{; \alpha ; \nu}-\delta_{\beta}^{\sigma} \delta_{\alpha}^{\tau}\left(h_{\sigma \tau}\right)_{; \nu}^{; \mu}\right],
$$

and we know that for the Weyl tensor $C_{\nu \mu \lambda}^{\mu}=0$. We then find that

$$
2 C_{\mu \nu \lambda \sigma} \delta \mathcal{F}_{3} C^{\mu \nu \lambda \sigma}=2\left(2 R_{\mu \nu} \mathcal{F}_{3} C_{\mu \nu \lambda \sigma}+\left(\mathcal{F}_{3} C_{\mu \nu \lambda \sigma}\right)_{; \mu ; \nu}\right) h_{\alpha \beta}
$$

So that

$$
\begin{aligned}
\delta S_{3}= & \int d^{4} x \sqrt{-g}\left[\frac{1}{2} g^{\alpha \beta} C^{\mu \nu \lambda \sigma} \mathcal{F}_{3} C_{\mu \nu \lambda \sigma}-2 C_{\rho \theta \psi}^{\alpha} \mathcal{F}_{3} C^{\beta \rho \theta \psi}\right. \\
& \left.+2\left[2 R_{\mu \nu} \mathcal{F}_{3} C_{\mu \nu \lambda \sigma}+\left(\mathcal{F}_{3} C_{\mu \nu \lambda \sigma}\right)_{; \mu ; \nu}\right]\right] h_{\alpha \beta}+C^{\mu \nu \lambda \sigma} \delta \mathcal{F}_{3} C_{\mu \nu \lambda \sigma} .
\end{aligned}
$$

To find the last term we continue as in the Ricci scalar case, only now using (20) instead of (21). We integrate by parts, repeatedly and simplify using (27) as well as the identity $C_{\mu \lambda \nu}^{\lambda}=0$ so that the final term reduces to follows

$$
\int d^{4} x \sqrt{-g} C^{\mu \nu \lambda \sigma} \delta \mathcal{F}_{3} C_{\mu \nu \lambda \sigma}=\int d^{4} x \sqrt{-g}\left(\Omega_{3}^{\alpha \beta}-\frac{1}{2} g^{\alpha \beta}\left(\Omega_{3 \gamma}^{\gamma}+\bar{\Omega}_{3}\right)+4 \Delta_{3}^{\alpha \beta}\right) h_{\alpha \beta},
$$

and we find the energy-momentum tensor to be

$$
\begin{gathered}
P_{3}^{\alpha \beta}=-g^{\alpha \beta} C^{\mu \nu \lambda \sigma} \mathcal{F}_{3}(\square) C_{\mu \nu \lambda \sigma}+4 C_{\mu \nu \sigma}^{\alpha} \mathcal{F}_{3}(\square) C^{\beta \mu \nu \sigma}-4\left(2 \nabla_{\mu} \nabla_{\nu}+R_{\mu \nu}\right) \mathcal{F}_{3}(\square) C^{\beta \mu \nu \alpha} \\
-2 \Omega_{3}^{\alpha \beta}+g^{\alpha \beta}\left(\Omega_{3 \gamma}^{\gamma}+\bar{\Omega}_{3}\right)-8 \Delta_{3}^{\alpha \beta}
\end{gathered}
$$

Here

$$
\begin{aligned}
& \Omega_{3}^{\alpha \beta}=\sum_{n=1}^{\infty} f_{3_{n}} \sum_{l=0}^{n-1} C_{\nu \lambda \sigma}^{\mu ; \alpha(l)} C_{\mu}^{\nu \lambda \sigma ; \beta(n-l-1)}, \quad \bar{\Omega}_{3}=\sum_{n=1}^{\infty} f_{3_{n}} \sum_{l=0}^{n-1} C_{\nu \lambda \sigma}^{\mu(l)} C_{\mu}^{\nu \lambda \sigma(n-l)}, \\
& \Delta_{3}^{\alpha \beta}=\frac{1}{2} \sum_{n=1}^{\infty} f_{3_{n}} \sum_{l=0}^{n-1}\left[C_{\sigma \mu}^{\lambda \nu(l)} C_{\lambda}^{(\beta|\sigma \mu| ; \alpha)(n-l-1)}-C_{\sigma \mu}^{\lambda \nu ;(\alpha(l)} C_{\lambda}^{\beta) \sigma \mu(n-l-1)}\right]_{; \nu} .
\end{aligned}
$$




\subsection{The Complete Field Equations}

Following from this we find the equation of motion for the full action $S$ in (1) to be a combination of $S_{0}, S_{1}, S_{2}$ and $S_{3}$ above

$$
\begin{aligned}
P^{\alpha \beta}=G^{\alpha \beta}+ & 4 G^{\alpha \beta} \mathcal{F}_{1}(\square) R+g^{\alpha \beta} R \mathcal{F}_{1}(\square) R-4\left(\nabla^{\alpha} \nabla^{\beta}-g^{\alpha \beta} \square\right) \mathcal{F}_{1}(\square) R \\
& -2 \Omega_{1}^{\alpha \beta}+g^{\alpha \beta}\left(\Omega_{1 \sigma}^{\sigma}+\bar{\Omega}_{1}\right)+4 R_{\mu}^{\alpha} \mathcal{F}_{2}(\square) R^{\mu \beta} \\
& -g^{\alpha \beta} R_{\nu}^{\mu} \mathcal{F}_{2}(\square) R_{\mu}^{\nu}-4 \nabla_{\mu} \nabla^{\beta}\left(\mathcal{F}_{2}(\square) R^{\mu \alpha}\right)+2 \square\left(\mathcal{F}_{2}(\square) R^{\alpha \beta}\right) \\
& +2 g^{\alpha \beta} \nabla_{\mu} \nabla_{\nu}\left(\mathcal{F}_{2}(\square) R^{\mu \nu}\right)-2 \Omega_{2}^{\alpha \beta}+g^{\alpha \beta}\left(\Omega_{2 \sigma}^{\sigma}+\bar{\Omega}_{2}\right)-4 \Delta_{2}^{\alpha \beta} \\
& -g^{\alpha \beta} C^{\mu \nu \lambda \sigma} \mathcal{F}_{3}(\square) C_{\mu \nu \lambda \sigma}+4 C_{\mu \nu \sigma}^{\alpha} \mathcal{F}_{3}(\square) C^{\beta \mu \nu \sigma} \\
& -4\left(R_{\mu \nu}+2 \nabla_{\mu} \nabla_{\nu}\right)\left(\mathcal{F}_{3}(\square) C^{\beta \mu \nu \alpha}\right)-2 \Omega_{3}^{\alpha \beta}+g^{\alpha \beta}\left(\Omega_{3 \gamma}^{\gamma}+\bar{\Omega}_{3}\right)-8 \Delta_{3}^{\alpha \beta} \\
& =T^{\alpha \beta},
\end{aligned}
$$

where $T^{\alpha \beta}$ is the stress energy tensor for the matter components in the universe and we have defined the following symmetric tensors:

$$
\begin{aligned}
& \Omega_{1}^{\alpha \beta}=\sum_{n=1}^{\infty} f_{1_{n}} \sum_{l=0}^{n-1} \nabla^{\alpha} R^{(l)} \nabla^{\beta} R^{(n-l-1)}, \quad \bar{\Omega}_{1}=\sum_{n=1}^{\infty} f_{1_{n}} \sum_{l=0}^{n-1} R^{(l)} R^{(n-l)}, \\
& \Omega_{2}^{\alpha \beta}=\sum_{n=1}^{\infty} f_{2_{n}} \sum_{l=0}^{n-1} R_{\nu}^{\mu ; \alpha(l)} R_{\mu}^{\nu ; \beta(n-l-1)}, \quad \bar{\Omega}_{2}=\sum_{n=1}^{\infty} f_{2_{n}} \sum_{l=0}^{n-1} R_{\nu}^{\mu(l)} R_{\mu}^{\nu(n-l)}, \\
& \Delta_{2}^{\alpha \beta}=\frac{1}{2} \sum_{n=1}^{\infty} f_{2_{n}} \sum_{l=0}^{n-1}\left[R_{\sigma}^{\nu(l)} R^{(\beta|\sigma| ; \alpha)(n-l-1)}-R_{\sigma}^{\nu ;(\alpha(l)} R^{\beta) \sigma(n-l-1)}\right]_{; \nu}, \\
& \Omega_{3}^{\alpha \beta}=\sum_{n=1}^{\infty} f_{3_{n}} \sum_{l=0}^{n-1} C_{\nu \lambda \sigma}^{\mu ; \alpha(l)} C_{\mu}^{\nu \lambda \sigma ; \beta(n-l-1)}, \bar{\Omega}_{3}=\sum_{n=1}^{\infty} f_{3_{n}} \sum_{l=0}^{n-1} C_{\nu \lambda \sigma}^{\mu(l)} C_{\mu}^{\nu \lambda \sigma(n-l)}, \\
& \Delta_{3}^{\alpha \beta}=\frac{1}{2} \sum_{n=1}^{\infty} f_{3_{n}} \sum_{l=0}^{n-1}\left[C_{\sigma \mu}^{\lambda \nu(l)} C_{\lambda}^{(\beta|\sigma \mu| ; \alpha)(n-l-1)}-C_{\sigma \mu}^{\lambda \nu ;(\alpha(l)} C_{\lambda}^{\beta) \sigma \mu(n-l-1)}\right]_{; \nu} .
\end{aligned}
$$

The trace equation is often particularly useful and below we provide it for the general action (1):

$$
\begin{aligned}
P=-R+12 & \square \mathcal{F}_{1}(\square) R+2 \square\left(\mathcal{F}_{2}(\square) R\right)+4 \nabla_{\mu} \nabla_{\nu}\left(\mathcal{F}_{2}(\square) R^{\mu \nu}\right) \\
& +2\left(\Omega_{1 \sigma}^{\sigma}+2 \bar{\Omega}_{1}\right)+2\left(\Omega_{2 \sigma}^{\sigma}+2 \bar{\Omega}_{2}\right)+2\left(\Omega_{3 \sigma}^{\sigma}+2 \bar{\Omega}_{3}\right)-4 \Delta_{2 \sigma}^{\sigma}-8 \Delta_{3 \sigma}^{\sigma} \\
& =T \equiv g_{\alpha \beta} T^{\alpha \beta} .
\end{aligned}
$$

It is worth noting that we have checked special cases of our result against previous work in sixth order gravity given in [24] and found them to be equivalent at least to the cubic order (see Appendix C for details). 


\section{Checks and Comparisons}

The mere complexity of the derived field equations for the Generalized Quadratic Curvature gravity warrants that we perform consistency tests and comparisons with previous known results. This is going to be the focus of this Section. We will start with testing Bianchi identities.

\subsection{Testing Bianchi Identities}

The stress energy tensor for any covariant gravitational action must satisfy what are called the Bianchi identities:

$$
P_{; \beta}^{\alpha \beta}=0 \text {. }
$$

Therefore, a strong test of the validity of the equation of motion (52) would be to take the covariant derivative and to check explicitly if it vanishes. Similar checks were also performed in [25] when looking at actions involving only the scalar curvature. In fact, it should be noted that the covariant derivative of each of $P_{0}^{\alpha \beta}, P_{1}^{\alpha \beta}, P_{2}^{\alpha \beta}, P_{3}^{\alpha \beta}$ should

vanish individually as they are independent of each other. Clearly $P_{0 ; \beta}^{\alpha \beta}=0$ as $G_{; \beta}^{\alpha \beta} \equiv 0$. Now, let us check whether the other expressions satisfy these conditions. We will focus on $P_{1}^{\alpha \beta}$. We have from (52):

$$
\begin{aligned}
& P_{1}^{\alpha \beta}=-4 G^{\alpha \beta} \mathcal{F}_{1}(\square) R+g^{\alpha \beta} R \mathcal{F}_{1}(\square) R-4\left(\nabla^{\alpha} \nabla^{\beta}-g^{\alpha \beta} \square\right) \mathcal{F}_{1}(\square) R \\
& +\sum_{n=1}^{\infty} f_{1_{n}} \sum_{l=0}^{n-1}\left\{-2 \nabla^{\alpha} R^{(l)} \nabla^{\beta} R^{(n-l-1)}+g^{\alpha \beta} \nabla^{\sigma} R^{(l)} \nabla_{\sigma} R^{(n-l-1)}+g^{\alpha \beta} R^{(l)} R^{(n-l)}\right\} .
\end{aligned}
$$

Next, we take the covariant derivative and cancel like terms

$$
\begin{aligned}
& P_{1 ; \beta}^{\alpha \beta}=4 R_{\sigma}^{\alpha} \nabla^{\sigma} \mathcal{F}_{1}(\square) R-4 \nabla^{\sigma} \nabla^{\alpha} \nabla_{\sigma} \mathcal{F}_{1}(\square) R+4 \nabla^{\alpha} \square \mathcal{F}_{1}(\square) R \\
& +\sum_{n=1}^{\infty} f_{1_{n}} \sum_{l=0}^{n-1}\left[\nabla_{\sigma} R^{(l)} \nabla^{\alpha} \nabla^{\sigma} R^{(n-l-1)}-\nabla^{\sigma} \nabla^{\alpha} R^{(l)} \nabla_{\sigma} R^{(n-l-1)}\right. \\
& \left.+R^{(l)} \nabla^{\alpha} R^{(n-l)}-\nabla^{\alpha} R^{(l)} R^{(n-l)}\right]
\end{aligned}
$$

Before using the following identity which comes from the general definition of a covariant derivative acting on a tensor

$$
\left[\nabla_{a}, \nabla_{b}\right] \lambda^{c}=R_{d a b}^{c} \lambda^{d}
$$

which gives us

$$
\nabla^{\sigma} \nabla^{\alpha} \nabla_{\sigma} \mathcal{F}_{1}(\square) R=\nabla^{\alpha} \square \mathcal{F}_{1}(\square) R+R_{\sigma}^{\alpha} \nabla^{\sigma} \mathcal{F}_{1}(\square) R
$$

Substituting, we find

$$
\begin{aligned}
& T_{; \beta}^{\alpha \beta}=\nabla^{\alpha} R \mathcal{F}_{1}(\square) R-R \nabla^{\alpha} \mathcal{F}_{1}(\square) R+\sum_{n=1}^{\infty} f_{1_{n}} \sum_{l=0}^{n-1}\left[\nabla_{\sigma} R^{(l)} \nabla^{\alpha} \nabla^{\sigma} R^{(n-l-1)}\right. \\
& \left.-\nabla^{\sigma} \nabla^{\alpha} R^{(l)} \nabla_{\sigma} R^{(n-l-1)}+R^{(l)} \nabla^{\alpha} R^{(n-l)}-\nabla^{\alpha} R^{(l)} R^{(n-l)}\right]
\end{aligned}
$$


Finally, using the following technical trick

$$
\sum_{n} \sum_{m} A^{(m)} B^{(n)}=\sum_{n} \sum_{m} A^{(n)} B^{(m)}
$$

along with the definition for $\mathcal{F}_{i}(\square)=\sum_{n=0}^{\infty} f_{i_{n}} \square^{n}$, we find that all terms cancel and so the Bianchi identity is satisfied. The same method can be applied to $S_{2}$ and $S_{3}$ by amending (64) so that the commutator acts upon tensors of different type with the addition of many more terms. Suffice to say, when all these terms are accounted for the Bianchi identity is indeed satisfied.

\subsection{Gravitation in a Weak-Field Limit}

Finally, we perform a last check of our equations involving linearized field equations around the Minkowski space-time that was derived for the action (11) in [10]. The algorithm is to look at fluctuations around the Minkowski space-time:

$$
g_{\mu \nu}=\eta_{\mu \nu}+h_{\mu \nu}
$$

$\eta^{\mu \nu}$ being the Minkowski metric. We then compute the field equations keeping terms only up to $\mathcal{O}(h)$ and compare with the equations derived in [10].

To accomplish this we need to compute all the relevant tensorial quantities up to linear order in $h_{\mu \nu}$. We start with the inverse of the metric:

$$
g^{\mu \nu}=\eta^{\mu \nu}-h^{\mu \nu} .
$$

Using (69) and (70) we then find the Christoffel symbols:

$$
\Gamma_{\mu \nu}^{\lambda}=\frac{1}{2} \eta^{\lambda \tau}\left(h_{\mu \tau, \nu}+h_{\tau \nu, \mu}-h_{\mu \nu, \tau}\right)+\mathcal{O}\left(h^{2}\right),
$$

where $\mathcal{O}\left(h^{2}\right)$ represents products of $h^{\mu \nu}$ which can be ignored in the weak-field limit. Substituting the expression for the Christoffel symbol into the general definitions for the Riemann tensor, Ricci tensor and curvature scalar we find the weak limit of these to be as follows:

$$
\begin{aligned}
& R_{\rho \mu \sigma \nu}=\frac{1}{2}\left(\partial_{\sigma} \partial_{\mu} h_{\rho \nu}+\partial_{\nu} \partial_{\rho} h_{\mu \sigma}-\partial_{\nu} \partial_{\mu} h_{\rho \sigma}-\partial_{\sigma} \partial_{\rho} h_{\mu \nu}\right), \\
& R_{\mu \nu}=\frac{1}{2}\left(\partial_{\sigma} \partial_{\mu} h_{\nu}^{\sigma}+\partial_{\nu} \partial_{\sigma} h_{\mu}^{\sigma}-\partial_{\nu} \partial_{\mu} h-\square h_{\mu \nu}\right), \\
& R=\partial_{\mu} \partial_{\nu} h^{\mu \nu}-\square h
\end{aligned}
$$

where $\square=\eta^{\mu \nu} \partial_{\mu} \partial_{\nu}$ and $h=\eta^{\mu \nu} h_{\mu \nu}$.

From (52), we find our equation of motion in the weak limit up to the linear order to be

$$
\begin{aligned}
P^{\alpha \beta}= & G^{\alpha \beta}-4\left(\nabla^{\alpha} \nabla^{\beta}-\eta^{\alpha \beta} \square\right) \mathcal{F}_{1} R-4 \nabla_{\mu} \nabla^{\beta}\left(\mathcal{F}_{2} R^{\mu \alpha}\right)+2 \square\left(\mathcal{F}_{2} R^{\alpha \beta}\right) \\
& +2 \eta^{\alpha \beta} \nabla_{\mu} \nabla_{\nu}\left(\mathcal{F}_{2} R^{\mu \nu}\right)-8 \nabla_{\mu} \nabla_{\nu} \mathcal{F}_{3}(\square) C^{\beta \mu \nu \alpha}
\end{aligned}
$$


One can then substitute equations (72) to (74) into the above field equation, in order to find the weak-limit equation of motion

$$
\begin{aligned}
P^{\alpha \beta}= & -\frac{1}{2}\left[1+2 \mathcal{F}_{2}(\square) \square+4 \mathcal{F}_{3}(\square) \square\right] \square h^{\alpha \beta} \\
& -\frac{1}{2}\left[-1-2 \mathcal{F}_{2}(\square) \square-4 \mathcal{F}_{3}(\square) \square\right] \partial_{\sigma}\left(\partial^{\alpha} h^{\sigma \beta}+\partial^{\beta} h^{\alpha \sigma}\right) \\
& -\frac{1}{2}\left[1-8 \mathcal{F}_{1}(\square) \square-2 \mathcal{F}_{2}(\square) \square+\frac{4}{3} \mathcal{F}_{3}(\square) \square\right]\left(\partial^{\beta} \partial^{\alpha} h+\eta^{\alpha \beta} \partial_{\mu} \partial_{\nu} h^{\mu \nu}\right) \\
& -\frac{1}{2}\left[-1+8 \mathcal{F}_{1}(\square) \square+2 \mathcal{F}_{2}(\square) \square-\frac{4}{3} \mathcal{F}_{3}(\square) \square\right] \eta^{\alpha \beta} \square h \\
& -\frac{1}{2}\left[8 \mathcal{F}_{1}(\square) \square+4 \mathcal{F}_{2}(\square) \square+\frac{8}{3} \mathcal{F}_{3}(\square) \square\right] \square^{-1} \nabla^{\alpha} \nabla^{\beta} \partial_{\mu} \partial_{\nu} h^{\mu \nu},
\end{aligned}
$$

where we have used the definition of the Weyl tensor (2) and substituted equations (72) to (74) in order to compute the final term. We may then rewrite this as

$$
\begin{gathered}
P^{\alpha \beta}=-\frac{1}{2}\left[a(\square) \square h^{\alpha \beta}+b(\square) \partial_{\sigma}\left(\partial^{\alpha} h^{\sigma \beta}+\partial^{\beta} h^{\sigma \alpha}\right)+c(\square)\left(\partial^{\alpha} \partial^{\beta} h+\eta^{\alpha \beta} \partial_{\mu} \partial_{\nu} h^{\mu \nu}\right)\right. \\
\left.+d(\square) \eta^{\alpha \beta} \square h+f(\square) \square^{-1} \partial^{\alpha} \partial^{\beta} \partial_{\mu} \partial_{\nu} h^{\mu \nu}\right],
\end{gathered}
$$

where we have defined the functions $a, b, c, d, f$ according to Ref. [10, 17]

$$
\begin{aligned}
& a(\square)=\left[1+2 \mathcal{F}_{2}(\square) \square+4 \mathcal{F}_{3}(\square) \square\right], \\
& b(\square)=\left[-1-2 \mathcal{F}_{2}(\square) \square-4 \mathcal{F}_{3}(\square) \square\right], \\
& c(\square)=\left[1-8 \mathcal{F}_{1}(\square) \square-2 \mathcal{F}_{2}(\square) \square+\frac{4}{3} \mathcal{F}_{3}(\square) \square\right], \\
& d(\square)=\left[-1+8 \mathcal{F}_{1}(\square) \square+2 \mathcal{F}_{2}(\square) \square-\frac{4}{3} \mathcal{F}_{3}(\square) \square\right], \\
& f(\square)=2\left[4 \mathcal{F}_{1}(\square) \square+2 \mathcal{F}_{2}(\square) \square+\frac{4}{3} \mathcal{F}_{3}(\square) \square\right],
\end{aligned}
$$

and we retrieve the following constraints [10, 17]

$$
\begin{aligned}
& a+b=0, \\
& c+d=0, \\
& b+c+f=0 .
\end{aligned}
$$

Let us make a few comments about the comparison: First, we point out the slight difference in the functions (78,82) as compared to [10, 17]. This is because we are using the "mostly positive" convention for the metric as opposed to "mostly negative" used in [10, 17]. Secondly, we have set $M_{p}=1$ as opposed to $M_{p}=2$ in [10, 17]. Thirdly, in [10, 17] the action and the $\mathcal{F}$ 's were defined using the Riemann tensor instead of the Weyl tensor that we are using here. We have also defined the functions (78, 82) in such a way so that the GR limit tends to 1 (i.e. $a(\square) \rightarrow 1$ when $\mathcal{F}_{i}(\square)=0$ ). Needless to say, once all these "convention" related differences are taken into account, (78,82) become consistent with the functions derived in [10, 17]. This concludes the consistency tests on our derived field equations. 


\section{Ghost \& Asympototically free Theories, a special subclass}

The linearized field equations derived in the last Subsection provides us with an insight about the quantum consistency and UV properties of such generalized gravitational theories. Essentially the functions $a(\square)$ through $f(\square)$ are related to the inverse propagators of the various metric degrees of freedom. Since poles in propagators provides us with physical degrees of freedom, it is possible to place simple criteria on these functions to ensure the theory doesn't contain unwanted degrees of freedom. For instance, if one does not want to introduce any new degrees of freedom apart from the massless graviton, then $f$ must vanish, which implies that one only has a single undetermined function: $a(\square)$ :

$$
a(\square)=c(\square)=-b(\square)=-d(\square) \Rightarrow 6 \mathcal{F}_{1}(\square)+3 \mathcal{F}_{2}(\square)+2 \mathcal{F}_{3}(\square)=0 .
$$

While several different $\mathcal{F}$ 's can satisfy the above relation, for the purpose of illustration let us consider the case when

$$
\mathcal{F}_{3}=0 \Rightarrow \mathcal{F}_{1}(\square)=-\frac{1}{2} \mathcal{F}_{2}(\square) \Rightarrow \mathcal{F}_{2}(\square)=\frac{a(\square)-1}{2 \square} .
$$

Further, the theory is ghost-free if $a(\square)$ is an entire function without any zeroes in the complex plane. Thus we obtain a special subclass of ghost-free quadratic curvature theories for the massless graviton given by

$$
S=\int d^{4} x \sqrt{-g}\left[\frac{R}{2}+R\left[\frac{a(\square)-1}{\square}\right] R-2 R_{\mu \nu}\left[\frac{a(\square)-1}{\square}\right] R^{\mu \nu}\right],
$$

with $a(0)=1$ ensuring that we recover GR in the low energy Newtonian limit.

A particularly simple class which mimics the stringy gaussian non-localities is given by

$$
a(\square)=e^{-\frac{\square}{M^{2}}} .
$$

By construction the above action contains only the graviton as physical degrees of freedom as in GR, but contains an exponentially damped propagator in the UV which, as was argued in [10], can have profound consequences for the gravitational singularities. For instance, one finds that if one considers the static weak field limit and try to derive the Newtonian potentials $\Phi, \Psi$ defined via

$$
d s^{2}=-(1+2 \Phi) d t^{2}+(1-2 \Psi) d \vec{r}^{2},
$$

then one finds that they do not diverge for a large class of entire functions, $a(\square)$. For $a(\square)=e^{-\square / M^{2}}, \Phi=\Psi \rightarrow$ to a constant [26] as $r \rightarrow 0$, i.e. the theory is asymptotically free.

One can read off the field equations for such theories from our expression (52) as

$$
\begin{aligned}
P^{\alpha \beta}=G^{\alpha \beta}+ & 4 G^{\alpha \beta}\left[\frac{e^{-\square / M^{2}}-1}{\square}\right] R+g^{\alpha \beta} R\left[\frac{e^{-\square / M^{2}}-1}{\square}\right] R \\
& -4\left(\nabla^{\alpha} \nabla^{\beta}-g^{\alpha \beta} \square\right)\left[\frac{e^{-\square / M^{2}}-1}{\square}\right] R-8 R_{\mu}^{\alpha}\left[\frac{e^{-\square / M^{2}}-1}{\square}\right] R^{\mu \beta}
\end{aligned}
$$




$$
\begin{aligned}
& +2 g^{\alpha \beta} R_{\nu}^{\mu}\left[\frac{e^{-\square / M^{2}}-1}{\square}\right] R_{\mu}^{\nu}+8 \nabla_{\mu} \nabla^{\beta}\left(\left[\frac{e^{-\square / M^{2}}-1}{\square}\right] R^{\mu \alpha}\right) \\
& -4 \square\left(\left[\frac{e^{-\square / M^{2}}-1}{\square}\right] R^{\alpha \beta}\right)-4 g^{\alpha \beta} \nabla_{\mu} \nabla_{\nu}\left(\left[\frac{e^{-\square / M^{2}}-1}{\square}\right] R^{\mu \nu}\right) \\
& +\sum_{n=1}^{\infty} \sum_{p=0}^{\infty}(-1)^{p+1} \frac{1}{(p+1) ! M^{2(p+1)}} \sum_{l=0}^{n-1}\left[-2 \nabla^{\alpha} R^{(l)} \nabla^{\beta} R^{(n-l-1)}\right. \\
& \left.+4 R^{\mu \nu ; \alpha(l)} R_{\mu \nu}^{; \beta(n-l-1)}+8\left[R_{\sigma}^{\nu(l)} R^{\beta \sigma ; \alpha(n-l-1)}-R_{\sigma}^{\nu ; \alpha(l)} R^{\beta \sigma(n-l-1)}\right]_{; \nu}\right\} \\
& +g^{\alpha \beta}\left(\nabla^{\sigma} R^{(l)} \nabla_{\sigma} R^{(n-l-1)}+R^{(l)} R^{(n-l)}-2 R^{\mu \nu ; \sigma(l)} R_{\mu \nu ; \sigma}^{(n-l-1)}\right. \\
& \left.\left.-2 R_{\nu}^{\mu(l)} R_{\mu}^{\nu(n-l)}\right)\right],
\end{aligned}
$$

where we have used (17) and (18) to find

$$
f_{1_{n}}=\sum_{n=0}^{\infty}(-1)^{n+1} \frac{1}{(n+1) ! M^{2(n+1)}} .
$$

\section{Conclusion}

To summarize, we have studied the classical equations of motion of the most general extension of Einstein's gravity including terms which are quadratic in curvatures. In particular these can contain an infinite set of higher derivative terms because we allow any number of covariant derivatives in our action. Our main result is the derivation of the field equations for these generalized theories. We have tested our results using the Bianchi identity and compared our results with known field equations for special subclasses of our starting action (11) as well as the weak-field limit that was derived in [10]. We then illustrated our results by specializing to a subclass of non-local ghost and asymptotically free theories of gravity.

What are the motivations behind looking into such non-local extensions of gravity? As mentioned in the introduction, GR suffers from a problem with infinities in the UV. For instance, classical singulatities appear in cosmological and black hole solutions. Now, it is known that higher derivative theories have better behaviour in the UV, but what conditions, must be set in order to formulate an alternative theory of gravity? We know from Lovelock's Theorem [2] that in order to preserve general covariance, we must accept higher than second derivatives of the metric *. In order to uphold unitarity, however, we must limit ourselves to theories of gravity which are free from ghosts. The action (88), for instance, describes a theory which is just that, namely, it is non-local, contains an infinite number of higher derivatives which help to avoid the problem of ghosts and is asymptotically free in the UV [10]. Needless to say the theory recovers the Newtonian gravitational potential in the IR. However, these statements have only been

* More technically, the theorem is true if we want a theory of gravity without giving up the metric tensor (graviton), four-dimensional space or $(0,2)$-tensor symmetry. 
robustly verified at the linearized level around the Minkowski space-time. The present work lays the groundwork for more revealing work around more general space-times and possibly incorporating nonlinear gravitational effects.

For example, one may then examine the results in the light of an expanding universe [11], such as in de Sitter space. Another step could be to derive the geodesic deviation equation and the Raychaudhuri equation for our theory of gravity in order to find a black hole solution that is free from singularities. As an aside, we remark that our formalisms and results may also find applications in gravitational research in other current areas, such as studies involving inflation theory or alternatives to dark energy.

\section{Acknowledgments}

Special thanks to Spyridon Talaganis for his helpful suggestions. A.C. is funded by STFC grant no ST/K50208X/1; A.K. is supported by an "FWO-Vlaanderen" postdoctoral fellowship and also supported in part by Belgian Federal Science Policy Office through the Interuniversity Attraction Pole P7/37, the "FWO-Vlaanderen" through the project G.0114.10N and the RFBR grant 11-01-00894; AM is supported by the Lancaster-Manchester-Sheffield Consortium for Fundamental Physics under STFC grant ST/J000418/1.

\section{Appendix A. Variation of the action}

\section{Appendix A.1. Background}

We have from the definitions of the Riemann and Ricci tensor

$$
\begin{aligned}
& \delta R_{\mu \sigma \nu}^{\lambda}=\left(\delta \Gamma_{\mu \nu}^{\lambda}\right)_{; \sigma}-\left(\delta \Gamma_{\mu \sigma}^{\lambda}\right)_{; \nu} \\
& \delta R_{\mu \nu}=\nabla_{\lambda} \delta \Gamma_{\mu \nu}^{\lambda}-\nabla_{\nu} \delta \Gamma_{\mu \lambda}^{\lambda} \\
& \delta \Gamma_{\mu \nu}^{\lambda}=\frac{1}{2}\left(h_{\nu ; \mu}^{\lambda}+h_{\mu ; \nu}^{\lambda}-h_{\mu \nu}^{; \lambda}\right)
\end{aligned}
$$

Expanding, we find

$$
\begin{aligned}
& \delta R_{\mu \sigma \nu}^{\lambda}=\frac{1}{2}\left(h_{\nu ; \mu ; \sigma}^{\lambda}-h_{\mu \nu ; \sigma}^{; \lambda}-h_{\sigma ; \mu ; \nu}^{\lambda}+h_{\mu \sigma ; \nu}^{; \lambda}\right) \\
& \delta R_{\mu \nu}=\frac{1}{2}\left(h_{\nu ; \mu ; \lambda}^{\lambda}+h_{\mu \lambda ; \nu}^{; \lambda}-\square h_{\mu \nu}-h_{; \mu ; \nu}\right)
\end{aligned}
$$

For simplicity later on we put these all in terms of the metric variation $h_{\alpha \beta}$

$$
\begin{aligned}
& \delta R_{\mu \nu \lambda \sigma}=\frac{1}{2}\left[\delta_{\lambda}^{\alpha} \delta_{\nu}^{\beta}\left(h_{\alpha \beta}\right)_{; \sigma ; \mu}-\delta_{\lambda}^{\alpha} \delta_{\mu}^{\beta}\left(h_{\alpha \beta}\right)_{; \sigma ; \nu}+\delta_{\mu}^{\alpha} \delta_{\sigma}^{\beta}\left(h_{\alpha \beta}\right)_{; \nu ; \lambda}-\delta_{\sigma}^{\alpha} \delta_{\nu}^{\beta}\left(h_{\alpha \beta}\right)_{; \mu ; \lambda}\right] \\
& \delta R_{\mu \nu}=\frac{1}{2}\left[\delta_{\nu}^{\beta}\left(h_{\alpha \beta}\right)_{; \mu}^{; \alpha}+\delta_{\mu}^{\beta}\left(h_{\alpha \beta}\right)_{; \nu}^{; \alpha}-\delta_{\mu}^{\alpha} \delta_{\nu}^{\beta} \square\left(h_{\alpha \beta}\right)-g^{\alpha \beta}\left(h_{\alpha \beta}\right)_{; \mu ; \nu}\right]
\end{aligned}
$$

Then we can find $\delta R$

$$
\delta R=\delta\left(g^{\mu \nu} R_{\mu \nu}\right)=\delta g^{\mu \nu} R_{\mu \nu}+g^{\mu \nu} \delta R_{\mu \nu}=-h_{\alpha \beta} R^{\alpha \beta}+g^{\mu \nu} \delta R_{\mu \nu}
$$


Ghost-free gravity

$$
\delta R=-h_{\alpha \beta} R^{\alpha \beta}+\left(h_{\alpha \beta}\right)^{; \alpha ; \beta}-g^{\alpha \beta} \square\left(h_{\alpha \beta}\right)
$$

where we have used the following notations

$$
h_{\mu \nu}=-h^{\alpha \beta} g_{\alpha \mu} g_{\beta \nu}, h=g^{\mu \nu} h_{\mu \nu}, h_{\mu \nu}=\delta g_{\mu \nu}, \quad \nabla_{\mu} S=S_{; \mu}
$$

In summary, for any tensor $S$ we have

$$
\begin{gathered}
\delta S_{\mu \nu \lambda \sigma}=\frac{1}{2}\left[\delta_{\lambda}^{\alpha} \delta_{\nu}^{\beta}\left(h_{\alpha \beta}\right)_{; \sigma ; \mu}-\delta_{\lambda}^{\alpha} \delta_{\mu}^{\beta}\left(h_{\alpha \beta}\right)_{; \sigma ; \nu}+\delta_{\mu}^{\alpha} \delta_{\sigma}^{\beta}\left(h_{\alpha \beta}\right)_{; \nu ; \lambda}-\delta_{\sigma}^{\alpha} \delta_{\nu}^{\beta}\left(h_{\alpha \beta}\right)_{; \mu ; \lambda}\right] \\
\delta S_{\mu \nu}=\frac{1}{2}\left[\delta_{\nu}^{\beta}\left(h_{\alpha \beta}\right)_{; \mu}^{; \alpha}+\delta_{\mu}^{\beta}\left(h_{\alpha \beta}\right)_{; \nu}^{; \alpha}-\delta_{\mu}^{\alpha} \delta_{\nu}^{\beta} \square\left(h_{\alpha \beta}\right)-g^{\alpha \beta}\left(h_{\alpha \beta}\right)_{; \mu ; \nu}\right] \\
\delta S=-h_{\alpha \beta} R^{\alpha \beta}+\left(h_{\alpha \beta}\right)^{; \alpha ; \beta}-g^{\alpha \beta} \square\left(h_{\alpha \beta}\right)
\end{gathered}
$$

and the Christoffel symbol in terms of $h_{\alpha \beta}$ is

$$
\delta \Gamma_{\mu \nu}^{\lambda}=\frac{1}{2}\left(g^{\lambda \alpha} \delta_{\nu}^{\beta} h_{\alpha \beta ; \mu}+g^{\lambda \alpha} \delta_{\mu}^{\beta} h_{\alpha \beta ; \nu}-\delta_{\mu}^{\alpha} \delta_{\nu}^{\beta} h_{\alpha \beta}^{; \lambda}\right)
$$

Appendix A.2. $\delta(\square) S$

Recall

$$
\square=g^{\mu \nu} \nabla_{\mu} \nabla_{\nu}
$$

Then we have

$$
\begin{aligned}
& \delta(\square) S=\delta g^{\mu \nu} S_{; \mu ; \nu}+g^{\mu \nu} \delta\left(\nabla_{\mu}\right) S_{; \nu}+g^{\mu \nu}\left[\delta\left(\nabla_{\nu}\right) S\right]_{; \mu} \\
& =-h_{\alpha \beta} S^{; \alpha ; \beta}+g^{\mu \nu} \delta\left(\nabla_{\mu}\right) S_{; \nu}+g^{\mu \nu}\left[\delta\left(\nabla_{\nu}\right) S\right]_{; \mu}
\end{aligned}
$$

From the general definition of the covariant derivative of a tensor we deduce the following $g^{\mu \nu} \delta\left(\nabla_{\mu}\right) S_{; \nu}=-g^{\mu \nu} \delta \Gamma_{\mu \nu}^{\lambda} S_{; \lambda}$

$g^{\mu \nu}\left[\delta\left(\nabla_{\nu}\right) S\right]_{; \mu}=0$

The last term vanishes in this case as $S$ is a scalar. This will not be true for $\delta(\square) S_{\mu \nu}$ and $\delta(\square) S_{\mu \nu \lambda \sigma}$. We then integrate by parts to find

$$
\delta(\square) S=-h_{\alpha \beta} S^{; \alpha ; \beta}+\frac{1}{2} g^{\alpha \beta} S_{; \lambda}\left(h_{\alpha \beta}\right)^{; \lambda}-S^{; \alpha}\left(h_{\alpha \beta}\right)^{; \beta}
$$

with

$$
\delta \Gamma_{\mu \nu}^{\lambda}=\frac{1}{2}\left[g^{\alpha \lambda} \delta_{\mu}^{\beta}\left(h_{\alpha \beta}\right)_{; \nu}+g^{\alpha \lambda} \delta_{\nu}^{\beta}\left(h_{\alpha \beta}\right)_{; \mu ;}-\delta_{\mu}^{\alpha} \delta_{\nu}^{\beta}\left(h_{\alpha \beta}\right)^{; \lambda}\right]
$$


Ghost-free gravity

Appendix A.3. $\delta(\square) S_{\mu \nu}$

$$
\begin{aligned}
& \delta(\square) S_{\mu \nu}=\delta g^{\lambda \sigma} S_{\mu \nu ; \lambda ; \sigma}+g^{\lambda \sigma} \delta\left(\nabla_{\lambda}\right) S_{\mu \nu ; \sigma}+g^{\lambda \sigma}\left[\delta\left(\nabla_{\sigma}\right) S_{\mu \nu}\right]_{; \lambda} \\
& =-h_{\alpha \beta} S_{\mu \nu}^{; \alpha ; \beta}+g^{\lambda \sigma} \delta\left(\nabla_{\lambda}\right) S_{\mu \nu ; \sigma}+g^{\lambda \sigma}\left[\delta\left(\nabla_{\sigma}\right) S_{\mu \nu}\right]_{; \lambda}
\end{aligned}
$$

From the general definition of the covariant derivative of a tensor we have

$g^{\lambda \sigma} \delta\left(\nabla_{\lambda}\right) S_{\mu \nu ; \sigma}=-\delta \Gamma_{\lambda \mu}^{\tau} S_{\tau \nu}^{; \lambda}-\delta \Gamma_{\lambda \nu}^{\tau} S_{\mu \tau}^{; \lambda}-g^{\lambda \sigma} \delta \Gamma_{\lambda \sigma}^{\tau} S_{\mu \nu ; \tau}$

$g^{\lambda \sigma} \nabla_{\lambda} \delta\left(\nabla_{\sigma}\right) S_{\mu \nu}=-\left(\delta \Gamma_{\lambda \mu}^{\tau}\right)^{; \lambda} S_{\tau \nu}-\delta \Gamma_{\lambda \mu}^{\tau} S_{\tau \nu}^{; \lambda}-\left(\delta \Gamma_{\lambda \nu}^{\tau}\right)^{; \lambda} S_{\mu \tau}-\delta \Gamma_{\lambda \nu}^{\tau} S_{\mu \tau}^{; \lambda}$

So that

$$
\delta(\square) S_{\mu \nu}=-h_{\alpha \beta} S_{\mu \nu}^{; \alpha ; \beta}-g^{\lambda \sigma} \delta \Gamma_{\lambda \sigma}^{\tau} S_{\mu \nu ; \tau}-\left(\delta \Gamma_{\lambda(\mu}^{\tau}\right)^{; \lambda} S_{\tau \nu)}-2 \delta \Gamma_{\lambda(\mu}^{\tau} S_{\tau \nu)}^{; \lambda}
$$

Expanding using $\delta \Gamma_{\mu \nu}^{\lambda}=\frac{1}{2}\left[g^{\alpha \lambda} \delta_{\mu}^{\beta}\left(h_{\alpha \beta}\right)_{; \nu}+g^{\alpha \lambda} \delta_{\nu}^{\beta}\left(h_{\alpha \beta}\right)_{; \mu ;}-\delta_{\mu}^{\alpha} \delta_{\nu}^{\beta}\left(h_{\alpha \beta}\right)^{; \lambda}\right]$, we have

$$
\begin{aligned}
& \delta(\square) S_{\mu \nu}=-h_{\alpha \beta} S_{\mu \nu}^{; \alpha ; \beta}-\left(h_{\alpha \beta}\right)^{; \beta} S_{\mu \nu}^{; \alpha}+\frac{1}{2} g^{\alpha \beta}\left(h_{\alpha \beta}\right)^{; \sigma} S_{\mu \nu ; \sigma} \\
& -\frac{1}{2}\left[\square\left(h_{\alpha \beta}\right) \delta_{(\mu}^{\beta} S_{\nu)}^{\alpha}-\left(h_{\alpha \beta}\right)^{; \tau ; \alpha} \delta_{(\mu}^{\beta} S_{\tau \nu)}+\left(h_{\alpha \beta}\right)_{;(\mu}^{; \beta} S_{\nu)}^{\alpha}\right] \\
& \left.-S_{(\nu}^{\alpha ; \beta} h_{\alpha \beta ; \mu)}-\delta_{(\mu}^{\beta} S_{\nu)}^{\alpha ; \lambda} h_{\alpha \beta ; \lambda}+\delta_{(\mu}^{\beta} S_{\tau \nu)}^{; \alpha} h_{\alpha \beta}^{; \tau}\right)
\end{aligned}
$$

Appendix A.4. $\delta(\square) S_{\mu \nu \lambda \sigma}$

From the definition of the D'Alembertian operator $\square=g^{\mu \nu} \nabla_{\mu} \nabla_{\nu}$, we have

$$
\begin{aligned}
& \delta(\square) S_{\mu \nu \lambda \sigma}=\delta g^{\kappa \tau} S_{\mu \nu \lambda \sigma ; \kappa ; \tau}+g^{\kappa \tau} \delta\left(\nabla_{\kappa}\right) S_{\mu \nu \lambda \sigma ; \tau}+g^{\kappa \tau}\left[\delta\left(\nabla_{\tau}\right) S_{\mu \nu \lambda \sigma}\right]_{; \kappa} \\
& =-h_{\alpha \beta} S_{\mu \nu \lambda \sigma}^{; \alpha ; \beta}+g^{\kappa \tau} \delta\left(\nabla_{\kappa}\right) S_{\mu \nu \lambda \sigma ; \tau}+g^{\kappa \tau}\left[\delta\left(\nabla_{\tau}\right) S_{\mu \nu \lambda \sigma}\right]_{; \kappa}
\end{aligned}
$$

and from the general definition of the covariant derivative of a tensor and treating $S_{\mu \nu \lambda \sigma ; \tau}$ as a $(0,5)$-tensor, we have

$$
\begin{gathered}
g^{\kappa \tau} \delta\left(\nabla_{\kappa}\right) S_{\mu \nu \lambda \sigma ; \tau}=-\delta \Gamma_{\kappa \mu}^{\rho} S_{\rho \nu \lambda \sigma}^{; \kappa}-\delta \Gamma_{\kappa \nu}^{\rho} S_{\mu \rho \lambda \sigma}^{; \kappa}-\delta \Gamma_{\kappa \lambda}^{\rho} S_{\mu \nu \rho \sigma}^{; \kappa}-\delta \Gamma_{\kappa \sigma}^{\rho} S_{\mu \nu \lambda \rho}^{; \kappa} \\
-g^{\kappa \tau} \delta \Gamma_{\kappa \tau}^{\rho} S_{\mu \nu \lambda \sigma ; \rho}
\end{gathered}
$$

and

$$
\begin{aligned}
g^{\kappa \tau}\left[\delta\left(\nabla_{\tau}\right) S_{\mu \nu \lambda \sigma}\right]_{; \kappa} & =\left[-\delta \Gamma_{\kappa \mu}^{\rho} S_{\rho \nu \lambda \sigma}-\delta \Gamma_{\kappa \nu}^{\rho} S_{\mu \rho \lambda \sigma}-\delta \Gamma_{\kappa \lambda}^{\rho} S_{\mu \nu \rho \sigma}-\delta \Gamma_{\kappa \sigma}^{\rho} S_{\mu \nu \lambda \rho}\right]^{; \kappa} \\
= & -\left(\delta \Gamma_{\kappa \mu}^{\rho}\right)^{; \kappa} S_{\rho \nu \lambda \sigma}-\delta \Gamma_{\kappa \mu}^{\rho} S_{\rho \nu \lambda \sigma}^{; \kappa}-\left(\delta \Gamma_{\kappa \nu}^{\rho}\right)^{; \kappa} S_{\mu \rho \lambda \sigma}-\delta \Gamma_{\kappa \nu}^{\rho} S_{\mu \rho \lambda \sigma}^{; \kappa} \\
& -\left(\delta \Gamma_{\kappa \lambda}^{\rho}\right)^{; \kappa} S_{\mu \nu \rho \sigma}-\delta \Gamma_{\kappa \lambda}^{\rho} S_{\mu \nu \rho \sigma}^{; \kappa}-\left(\delta \Gamma_{\kappa \sigma}^{\rho}\right)^{; \kappa} S_{\mu \nu \lambda \rho}-\delta \Gamma_{\kappa \sigma}^{\rho} S_{\mu \nu \lambda \rho}^{; \kappa}
\end{aligned}
$$

So that

$$
\begin{aligned}
& \delta(\square) S_{\mu \nu \lambda \sigma}=-h_{\alpha \beta} S_{\mu \nu \lambda \sigma}^{; \alpha ; \beta}-g^{\kappa \tau} \delta \Gamma_{\kappa \tau}^{\rho} S_{\mu \nu \lambda \sigma ; \rho} \\
& -\left[\left(\delta \Gamma_{\kappa \mu}^{\rho}\right)^{; \kappa} S_{\rho \nu \lambda \sigma}+\left(\delta \Gamma_{\kappa \nu}^{\rho}\right)^{; \kappa} S_{\mu \rho \lambda \sigma}+\left(\delta \Gamma_{\kappa \lambda}^{\rho}\right)^{; \kappa} S_{\mu \nu \rho \sigma}+\left(\delta \Gamma_{\kappa \sigma}^{\rho}\right)^{; \kappa} S_{\mu \nu \lambda \rho}\right] \\
& -2\left[\delta \Gamma_{\kappa \mu}^{\rho} S_{\rho \nu \lambda \sigma}^{; \kappa}+\delta \Gamma_{\kappa \nu}^{\rho} S_{\mu \rho \lambda \sigma}^{; \kappa}+\delta \Gamma_{\kappa \lambda}^{\rho} S_{\mu \nu \rho \sigma}^{; \kappa}+\delta \Gamma_{\kappa \sigma}^{\rho} S_{\mu \nu \lambda \rho}^{; \kappa}\right]
\end{aligned}
$$


Then, using $\delta \Gamma_{\mu \nu}^{\lambda}=\frac{1}{2}\left[g^{\alpha \lambda} \delta_{\mu}^{\beta}\left(h_{\alpha \beta}\right)_{; \nu}+g^{\alpha \lambda} \delta_{\nu}^{\beta}\left(h_{\alpha \beta}\right)_{; \mu ;}-\delta_{\mu}^{\alpha} \delta_{\nu}^{\beta}\left(h_{\alpha \beta}\right)^{; \lambda}\right]$, and the Bianchi identities, we find

$$
\begin{aligned}
& \delta(\square) S_{\mu \nu \lambda \sigma}=-h_{\alpha \beta} S_{\mu \nu \lambda \sigma}^{; \alpha ; \beta}-\left(h_{\alpha \beta}\right)^{; \beta} S_{\mu \nu \lambda \sigma}^{; \alpha}+\frac{1}{2} h^{; \tau} S_{\mu \nu \lambda \sigma ; \tau} \\
& -\frac{1}{2}\left[g^{\alpha \tau}\left(h_{\alpha \beta}\right)_{; \mu}^{; \beta} S_{\tau \nu \lambda \sigma}+g^{\alpha \tau}\left(h_{\alpha \beta}\right)_{; \nu}^{; \beta} S_{\mu \tau \lambda \sigma}+g^{\alpha \tau}\left(h_{\alpha \beta}\right)_{; \lambda}^{; \beta} S_{\mu \nu \tau \sigma}+g^{\alpha \tau}\left(h_{\alpha \beta}\right)_{; \sigma}^{; \beta} S_{\mu \nu \lambda \tau}\right] \\
& -\left[g^{\alpha \tau}\left(h_{\alpha \beta}\right)_{; \mu} S_{\tau \nu \lambda \sigma}^{; \beta}+g^{\alpha \tau}\left(h_{\alpha \beta}\right)_{; \nu} S_{\mu \tau \lambda \sigma}^{; \beta}+g^{\alpha \tau}\left(h_{\alpha \beta}\right)_{; \lambda} S_{\mu \nu \tau \sigma}^{; \beta}+g^{\alpha \tau}\left(h_{\alpha \beta}\right)_{; \sigma} S_{\mu \nu \lambda \tau}^{; \beta}\right]
\end{aligned}
$$

\section{Appendix B. Comparison with sixth-order Gravity}

Field equations for the most general gravitational action up to sixth order in derivatives were derived in [24]. This is the only action known to us which contains $\square$ operators acting on Weyl tensors, and hence it is useful to check our results against field equations given in [24]. For our action (1), this would mean keeping terms only up to a single box, i.e. $f_{\text {In }}=0$ for $n \geq 2$. More explicitly the field equations read as follows

$$
\begin{aligned}
& P^{\alpha \beta}=G^{\alpha \beta}+4 G^{\alpha \beta}\left(f_{10}+f_{11} \square\right) R+g^{\alpha \beta} R\left(f_{10}+f_{11} \square\right) R \\
& -4\left(\nabla^{\alpha} \nabla^{\beta}-g^{\alpha \beta} \square\right)\left(f_{10}+f_{11} \square\right) R-2 f_{11} \nabla^{\alpha} R \nabla^{\beta} R \\
& +g^{\alpha \beta} f_{11}\left(\nabla^{\gamma} R \nabla_{\gamma} R+R \square R\right)+4 R_{\mu}^{\alpha}\left(f_{20}+f_{21} \square\right) R^{\mu \beta}-g^{\alpha \beta} R_{\nu}^{\mu}\left(f_{20}\right. \\
& \left.+f_{21} \square\right) R_{\mu}^{\nu}-4 \nabla_{\mu} \nabla^{\beta}\left(\left(f_{20}+f_{21} \square\right) R^{\mu \alpha}\right)+2 \square\left(\left(f_{20}+f_{21} \square\right) R^{\alpha \beta}\right) \\
& +2 g^{\alpha \beta} \nabla_{\mu} \nabla_{\nu}\left(\left(f_{20}+f_{21} \square\right) R^{\mu \nu}\right)-2 f_{21} \nabla^{\alpha} R_{\nu}^{\mu} \nabla^{\beta} R_{\mu}^{\nu} \\
& +g^{\alpha \beta} f_{21}\left(\nabla^{\gamma} R_{\nu}^{\mu} \nabla_{\gamma} R_{\mu}^{\nu}+R_{\nu}^{\mu} \square R_{\mu}^{\nu}\right)-4 f_{21}\left(R_{\mu}^{\nu} \nabla^{\alpha} R^{\beta \mu}-R^{\beta \mu} \nabla^{\alpha} R_{\mu}^{\nu}\right)_{; \nu} \\
& -g^{\alpha \beta} C^{\mu \nu \lambda \sigma}\left(f_{30}+f_{31} \square\right) C_{\mu \nu \lambda \sigma}+4 C_{\mu \nu \sigma}^{\alpha}\left(f_{30}+f_{31} \square\right) C^{\beta \mu \nu \sigma} \\
& -4\left(R_{\mu \nu}+2 \nabla_{\mu} \nabla_{\nu}\right)\left(\left(f_{30}+f_{31} \square\right) C^{\beta \mu \nu \alpha}\right)-2 f_{31} \nabla^{\alpha} C^{\mu \nu \rho \gamma} \nabla^{\beta} C_{\mu \nu \rho \gamma} \\
& +g^{\alpha \beta} f_{31}\left(\nabla^{\alpha} C^{\mu \nu \rho \gamma} \nabla^{\beta} C_{\mu \nu \rho \gamma}+C^{\mu \nu \rho \gamma} \square C_{\mu \nu \rho \gamma}\right) \\
& -8 f_{31}\left(C^{\gamma \nu}{ }_{\rho \mu} \nabla^{\alpha} C_{\gamma}{ }^{\beta \rho \mu}-C_{\gamma}{ }^{\beta \rho \mu} \nabla^{\alpha} C_{\rho \mu}^{\gamma \nu}\right)_{; \nu}
\end{aligned}
$$

Unfortunately, this expression cannot be directly compared with the terms in [24], most specifically in their equation (2.23) because in 24] several identities (given in their Section 5) were used to convert quadratic curvature terms with a $\square$ to cubic in curvature without a $\square$. The full matching of the expressions therefore becomes a rather arduous task which is not particularly illuminating and we do not include this here. Nevertheless we have explicitly checked that expansions up to cubic order around the Minkowski background perfectly match.

[1] Clifford M W 1986 Was Einstein right?: putting general relativity to the test, New York: Basic Books

[2] Lovelock D 1975 Tensors differential forms and variational principles, Wiley-Interscience, New York

[3] Stelle K S 1977 "Renormalization of Higher Derivative Quantum Gravity," Phys. Rev. D 16, 953

[4] Stelle K S 1978 "Classical Gravity with Higher Derivatives," Gen. Rel. Grav. 9, 353

[5] Quandt I and Schmidt H J 1991 "The Newtonian limit of fourth and higher order gravity," Astron. Nachr. 31297 gr-qc/0109005.

[6] Biswas T, Mazumdar A and Siegel W 2006 Bouncing universes in string-inspired gravity, JCAP 0603009 hep-th/0508194 
[7] Tomboulis E T 1997 Superrenormalizable gauge and gravitational theories, hep-th/9702146

[8] Biswas T, Koivisto T and Mazumdar A 2010 Towards a resolution of the cosmological singularity in non-local higher derivative theories of gravity, JCAP 1011008 arXiv:1005.0590 [hep-th]];

Biswas T, Koshelev A S, Mazumdar A and Vernov S Yu 2012 Stable bounce and inflation in non-local higher derivative cosmology, JCAP 1208, 024 [arXiv:1206.6374 [astro-ph.CO]]

[9] Barnaby N, Biswas T and Cline J M 2007 p-adic Inflation, JHEP 0704056 hep-th/0612230; Aref'eva I Ya and Koshelev A S 2008 Cosmological Signature of Tachyon Condensation, JHEP 0809068 arXiv:0804.3570 [hep-th]];

[10] Biswas T, Gerwick E, Koivisto T and Mazumdar A 2012 "Towards singularity and ghost free theories of gravity," Phys. Rev. Lett. 108, 031101 arXiv:1110.5249 [gr-qc]]

[11] Koshelev A S 2013 Class. Quant. Grav. 30155001 [arXiv:1302.2140 [astro-ph.CO]]

[12] Khoury J 2007 Fading gravity and self-inflation, Phys. Rev. D 76, 123513 [hep-th/0612052];

Biswas T, Brandenberger R, Mazumdar A and Siegel W, 2007 Non-perturbative gravity, Hagedorn bounce and CMB, JCAP 0712011 hep-th/0610274];

Dimitrijevic I, Dragovich B, Grujic J and Rakic Z 2013 New Cosmological Solutions in Nonlocal Modified Gravity, arXiv:1302.2794 [gr-qc];

Barvinsky A O and Gusev Y V 2012 New representation of the nonlocal ghost-free gravity theory, arXiv:1209.3062 [hep-th];

Modesto L and Tsujikawa S 2013 Non-local massive gravity, arXiv:1307.6968 [hep-th];

Briscese F, Modesto L and Tsujikawa S 2013 Super-renormalizable or finite completion of the Starobinsky theory, arXiv:1308.1413 [hep-th]

[13] Krasnov K 2006 Renormalizable Non-Metric Quantum Gravity?, hep-th/0611182,

[14] Moffat J W 2011 Ultraviolet Complete Quantum Gravity, Eur. Phys. J. Plus 12643 arXiv:1008.2482 [gr-qc]];

Modesto L 2012 Super-renormalizable Quantum Gravity, Phys. Rev. D 86044005 arXiv:1107.2403 [hep-th]];

Calcagni G, Modesto L and Nicolini P 2013 Super-accelerating bouncing cosmology in asymptotically-free non-local gravity, arXiv:1306.5332 [gr-qc];

Neupane I P 2002 Localized gravity with higher curvature terms, Class. Quant. Grav. 195507 hep-th/0106100;

Neupane I P 2000 Consistency of higher derivative gravity in the brane background, JHEP 0009 040 hep-th/0008190]

[15] Witten E 1986 Noncommutative Geometry and String Field Theory, Nucl. Phys. B 268 253;

Freund P G O and Olson M 1987 Nonarchimedean Strings, Phys. Lett. B 199 186;

Vladimirov V S, Volovich I V, Zelenov I V 1994 p-adic Analysis and Mathematical Physics, Singapore: World Scientific

Biswas T, Grisaru M and Siegel W 2005 Nucl. Phys. B 708, 317 hep-th/0409089

[16] Van Nieuwenhuizen P 1973 "On ghost-free tensor lagrangians and linearized gravitation," Nucl. Phys. B 60, 478

[17] Biswas T, Koivisto T and Mazumdar A 2013 "Nonlocal theories of gravity: the flat space propagator," arXiv:1302.0532 [gr-qc]

[18] Ostrogradski M 1850 Memoires sur les equations differentielles relatives au probleme des isoperimetres, Mem. Ac. St. Petersburg VI 4385

[19] Barnaby N and Kamran N 2008 Dynamics with infinitely many derivatives: The Initial value problem, JHEP 0802008 arXiv:0709.3968 [hep-th]];

Barnaby N 2011 A New Formulation of the Initial Value Problem for Nonlocal Theories, Nucl. Phys. B 8451 arXiv:1005.2945 [hep-th]]

[20] Starobinsky A A 1980 Phys. Lett. B 91, 99 
[21] Mazumdar A and Rocher J 2011 Particle physics models of inflation and curvaton scenarios, Phys. Rept. 49785 [arXiv:1001.0993 [hep-ph]]

[22] Biswas T and Mazumdar A 2013 arXiv:1304.3648 [hep-th]

[23] Deser S and Woodard R P 2013 arXiv:1307.6639 [astro-ph.CO]

[24] Decanini Y and Folacci A 2007 Class. Quant. Grav. 244777 [arXiv:0706.0691 [gr-qc]]

[25] Schmidt H J 1990 "Variational derivatives of arbitrarily high order and multiinflation cosmological models," Class. Quant. Grav. 7, 1023

[26] Nesseris S and Mazumdar A 2009 Phys. Rev. D 79, 104006 arXiv:0902.1185 [astro-ph.CO]] 